\title{
Cardiopulmonary test in elderly: feasible, safe and useful
}

\author{
Angela Beatrice Scardovi · Renata De Maria
}

Received: 22 May 2009/Accepted: 27 May 2009/Published online: 2 July 2009

(C) SIMI 2009

We thank Dr. Donadini and Colleagues for the comments [1] to our paper entitled "Prognostic value of brain natriuretic peptide and enhanced ventilatory response to exercise in patients with chronic heart failure." [2] In this paper, we demonstrated the additive value of $\mathrm{CPx}$ in the prognostic stratification of HF in a "young elderly" population (mean age 68 years) of 156 consecutive patients. In a recently published paper [3], we demonstrated the safety and feasibility of CPx in the elderly. Our conclusions in that work were that this population normally excluded from CPx can benefit in terms of improved prognostic stratification from this test. Even in the present report, we confirm that a large proportion of elderly patients can undergo CPx: about one-fourth of patients (48 subjects, $24 \%$ ) were in fact excluded from the study for clinical contraindications, the main reasons for exclusion being orthopaedic problems (38 subjects, 79\%), cardiac conditions in six patients (severe aortic stenosis 3, mitral stenosis 1 , recent onset atrial fibrillation 2 and severe chronic lung disease in 3). Furthermore, we excluded four subjects with severe kidney dysfunction that might impact on BNP concentrations, while six patients refused to undergo the procedure.

Moreover, Dr. Donadini invites to consider with caution the results of BNP assessment in this population due to the possible high prevalence of pulmonary hypertension (from thromboembolism or chronic pulmonary disease) or chronic renal impairment. Both situations were reasons for exclusion
[2] so that we believe that BNP assessment could be considered a reliable parameter in the studied population. On the other side, the demonstration of high BNP in a screening strategy in the elderly must be followed by an echocardiogram, which is discriminating for a possible false positive.

The small HR of $V \mathrm{E} / V \mathrm{CO}_{2}$ slope 1.07 (95\% CI 1.011.13) results from the inclusion of the continuous slope value in the model: each one-point slope increase determines a $7 \%$ increase in mortality. Conversely, when EVR $\left(V \mathrm{E} / \mathrm{VCO}_{2}\right.$ slope $\geq 35$ ) is included as a discrete value in the model, we observe a fourfold increase in the risk of death (HR 4.02, 95\% CI 1.17-10.3), as shown in Fig. 2 of Ref. [2].

Conflict of interest statement The authors declare that they have no conflict of interest related to the publication of this manuscript.

\section{References}

1. Donadini MP, Squizzato A, Dentali F, Ageno W (2009) Prognostic stratification of chronic heart failure in elderly population: are cardiopulmonary tests and BNP really valuable. Intern Emerg Med. doi:10.1007/s11739-009-0247-2

2. Scardovi AB, De Maria R, Celestini A et al (2008) Prognostic value of brain natriuretic peptide and enhanced ventilatory response to exercise in patients with chronic heart failure. Intern Emerg Med 3:331-337

3. Scardovi AB, Coletta C, De Maria R et al (2007) The cardiopulmonary exercise test is safe and reliable in elderly patients with chronic heart failure. J Cardiovasc Med 8:608-612

\footnotetext{
A. B. Scardovi $(\square)$

Department of Cardiology, S. Spirito Hospital,

Lungotevere in Sassia 3, 00100 Rome, Italy

e-mail: ab.scardovi@libero.it

R. De Maria

CNR Clinical Physiology Institute, Milan, Italy
} 